\title{
APLICAÇÃO DO ÍNDICE DE ESPECIFICIDADE DE HÁBITAT EM CHLOROCOCCALES FITOPLANCTÔNICAS, COMO INDICADOR DE QUALIDADE AMBIENTAL EM RIOS COSTEIROS: ESTUDO DE CASO DO RIO ITAJAÍ-MIRIM, SC, BRASIL
}

\author{
MIRANDA, A.L.B. ${ }^{1,2}$; BEAUMORD, A. C. ${ }^{2}$ \& R.S.P. MELLO \\ ${ }^{1}$ Recém-doutor CNPq; burliga@cttmar.univali.br \\ ${ }^{2}$ Centro de Ciências Tecnológicas da Terra e do Mar, CTTMar, Universidade do Vale \\ do Itajaí, Univali, C.P. 360, Itajaí, SC 88302-202 \\ ${ }^{3}$ Instituto do Meio Ambiente, Pontifícia Universidade Católica do Rio Grande do Sul, \\ PUCRS. Av. Ipiranga 6681, Porto Alegre, RS 90619-900
}

\begin{abstract}
RESUMO
Indicadores de qualidade ambiental, baseados em índices de comunidades biológicas, vêm sendo amplamente utilizados em programas de diagnósticos e monitoramentos ambientais. Novos índices de comunidades vêem sendo propostos com a finalidade de se refinar mais estas aplicações. Neste trabalho foi aplicado o índice de especificidade de hábitat buscando-se avaliar a especificidade das espécies e dos gêneros de Chlorococcales em relação ao hábitat, e a contribuição da especificidade relativa do hábitat, na escala de paisagem. Foi realizado um levantamento qualitativo das Chlorococcales fitoplanctônicas em 5 estações amostrais no trecho médio do rio Itajaí-Mirim, entre os municípios de Brusque e Botuverá, de forma a verificar os padrões deste grupo durante a estação de verão. Foram identificadas 31 espécies, distribuídas em 12 gêneros. O maior valor do índice de especificidade foi obtido na estação amostral localizada no município de Botuverá, e o menor índice na estação localizada na área urbana de Brusque. Nesta escala o índice de especificidade genérica demonstrou mais claramente a formação de grupos de especificidade de hábitat.
\end{abstract}

Palavras-chave: Chlorococcales, rios costeiros, especificidade de hábitat, riqueza específica

\section{UTILIZATION OF THE HABITAT SPECIFICITY INDEX IN PHYTOPLANKTON CHLOROCOCCALES AS AN ENVIRONMENTAL QUALITY INDICATOR IN COASTAL RIVERS: CASE STUDY OF THE ITAJAII-MIRIM RIVER, SC, BRAZIL}

\begin{abstract}
Environmental quality indicators based on biological community indexes have been largely used in environmental characterization and monitoring programs. New community indexes have been proposed in order to refine such applications. In this work, we used the habitat specificity index to assess the specificity of the species and genera of Chlorococcales related to habitat, and the contribution of the relative habitat specificity, at the landscape scale. Thus, we conducted a survey in 5 sample sites in the medium stretch of the Itajaí-Mirim River, between the counties of Brusque e Botuverá, in order to investigated the patterns of phytoplankton Chlorococcales, in the summer season. It was identified 31 species belonging to 12 genera. The highest habitat specificity was found in the sample site located in Botuverá County (pristine), whereas the lowest, in the
\end{abstract}


Brusque County (disturbed). At such scale, the generic specificity index showed clearly groups of habitat specificity.

Key-words: Chlorococcales, coastal rivers, habitat specificity, species richness.

\section{INTRODUÇÃO}

A utilização de índices bióticos de comunidades, expressando de forma numérica um conjunto de dados sobre a composição da flora ou fauna, de forma a avaliar os efeitos da qualidade ambiental tem chamado a atenção dos cientistas desde o início do século vinte (Karr et al., 1999). O índice de diversidade biótica mais simples de um hábitat, expresso pelo número de espécies é a riqueza de espécies. Entretanto, a comparação de hábitats através da riqueza específica pode não refletir a importância relativa das espécies no conjunto de hábitats na escala de paisagem. Problemas como o da especificidade de espécies ao hábitat são detectados. Espécies generalistas ocorrem em muitos ou na maioria dos hábitats e, por outro lado, outras espécies podem ser restritas a um ou a poucos tipos de hábitats (Forman, 1995).

Um dos objetivos mais comuns na análise de comunidades é detectar e descrever o valor de diferentes espécies para indicar condições ambientais. O índice proposto por Wagner \& Edwards (2001) foi desenvolvido para estudos de ecologia de paisagem, com o intuito de verificar o valor potencial de uma área para conservação, baseado no argumento de que é mais importante saber quais espécies estão presentes em uma área do que quantas estão presentes. Estes índices fornecem um valor quantitativo do grau de afinidade (especificidade) da espécie em relação ao hábitat e ainda contabilizam a contribuição relativa da especificidade do hábitat na paisagem. Isto é, quanto maior a especificidade biológica de um hábitat, maior é a sua contribuição relativa para o conjunto da biodiversidade na escala da paisagem. Junto a esta questão, está o grau de diferenciação entre os hábitats, associado à distribuição das espécies em relação às características ambientais (Dufrene \& Legendre, 1997).

Se as diferenças ambientais são definidas como grupos de unidades amostrais ou hábitats, o método de cálculo de especificidade fornece valores para as espécies para uma dada tipologia ambiental, informando sobre a concentração de abundância das espécies em um grupo particular e a proporção de ocorrência de uma espécie em um grupo particular. Os grupos de unidades amostrais são freqüentemente definidos por variáveis ambientais categóricas, níveis de perturbação, tratamentos experimentais, dados de presença e ausência de uma espécie-alvo, ou por tipos de hábitats.

Vários índices bióticos estão sendo utilizados atualmente com o propósito de monitorar a qualidade ambiental dos rios, utilizando diversos grupos de algas (Kelly \& Whitton, 1998). Na análise preliminar do fitoplâncton no trecho médio do rio Itajaí-Mirim, foi verificada a dominância do grupo das Chlorococcales. Este grupo é referido como indicador de qualidade de águas mesoeutróficas a eutróficas, e mesososapróbias, ou seja, poluição orgânica moderada (Komárek \& Fott, 1983).

Este trabalho teve como objetivo a aplicação e avaliação do índice de especificidade de hábitat (Wagner \& Edwards, 2001), com base no levantamento qualitativo das Chlorococcales fitoplanctônicas no trecho médio do rio Itajaí-Mirim, durante a estação de verão.

\section{Descrição da área}

O rio Itajaí-Mirim, localizado entre as coordenadas Latitude $26^{\circ} 53^{\prime} 17,1^{\prime \prime} \mathrm{S}$ e $26^{\circ}$ 56 ' 05,1 'S; Longitude $48^{\circ} 40^{\prime} 57,8^{\prime \prime} \mathrm{W}$ e $48^{\circ} 44^{\prime}$ 12,4 "W, é um afluente da margem direita do rio 
Itajaí-Açu, sendo seu maior tributário, possuindo uma bacia com área aproximada de $1.700 \mathrm{~m} \mathrm{~km}^{2}$ e perímetro de aproximadamente 300 km, com uma extensão aproximada em seu leito principal de $176 \mathrm{~km}$. Esta bacia engloba integralmente os municípios de Vidal Ramos, Presidente Nereu, Botuverá, Guabiruba e Brusque, em sua totalidade, e em parte, os de Itajaí, Camboriú, Ilhota e Gaspar, totalizando 9 municípios. A bacia de drenagem é composta pelo rio principal e 17 tributários. Os municípios dos cursos alto e médio da bacia, dentre eles Botuverá, caracterizam-se por apresentar o setor primário como sua principal atividade econômica; enquanto que os municípios da parte baixa, como é o caso de Brusque, têm suas atividades econômicas voltadas para o setor secundário, com alto grau de industrialização. Desta forma, a qualidade ambiental dos cursos d'água à montante da bacia é bem superior à dos cursos à jusante (Riffel \& Beaumord, 2002; Silva \& Beaumord, 2002).

\section{MATERIAL E MÉTODOS}

Foi realizado o levantamento qualitativo das Chlorococcales fitoplanctônicas em 5 estações amostrais no trecho médio rio ItajaíMirim, entre os municípios de Brusque e Botuverá, em 10/02/2004. As estações amostrais foram consideradas como hábitats distintos, com base no conceito de rio contínuo (Vannote, 1989) formado por múltiplos hábitats no gradiente longitudinal do rio. A estação amostral 1 situa-se no município de Botuverá, na localidade Barra do Ouro. É uma área rural, com predomínio de plantios de fumo e floresta nativa em diversos estágios de sucessão. A estação 2 situa-se também no município de Botuverá, na localidade de São João Batista, com o predomínio de floresta nativa e pecuária. A estação 3 situa-se à montante da área urbana de Brusque, caracterizando-se como uma área mista entre atividades rurais (pecuária) e industriais (têxtil). A estação 4 está localizada em área urbana, mais precisamente no centro da cidade de Brusque, com indústrias têxteis e com vias de trânsito próximas às margens do rio, além de receber esgotos domésticos. A estação 5 localiza-se a jusante da cidade de Brusque, sendo também uma área mista, sob influência de efluentes industriais e esgotos domésticos, e ocupação rural nas margens do rio (Figura 1).

O método de coleta do fitoplâncton foi por concentração com rede de plâncton com abertura de malha de $25 \mathrm{~mm}$. A passagem da rede foi realizada durante 5 minutos longitudinalmente, em água subsuperficial, aproximadamente a $2 \mathrm{~m}$ de distância da margem.

$\mathrm{Na}$ análise qualitativa do fitoplâncton utilizou-se microscópio binocular em 1000 x, sendo os indivíduos identificados em cada estação amostral até cessarem as espécies novas. A bibliografia básica utilizada para a identificação dos táxons foi de Komárek \& Fott (1983); Rosa \& Miranda-Kiesslich (1988); Rosa \& Miranda-Kiesslich (1989).

O método de cálculo da especificidade pressupõe que existam, a priori, dois ou mais grupos de unidades amostrais, e que a abundância das espécies estejam registradas em cada uma das unidades amostrais. Para cada espécie, os seguintes passos foram realizados: calcula-se a abundância proporcional de uma espécie particular em um grupo particular, em relação à abundância daquela espécie em todos os grupos (hábitats), sendo o resultado expresso em porcentagem. A especificidade de um determinado hábitat é a soma das especificidades das espécies neste hábitat.

A partir dos dados sobre a comunidade de Chlorococcales fitoplanctônicas, com 31 espécies distribuídas em 5 u.a. (Estações 1, 2, 3, 4 e 5), foi gerada uma matriz de presença e ausência e calculados os índices de especificidade de espécies, de gêneros e das áreas (hábitats), conforme proposto por Wagner \& Edwards (2001). 


$$
\begin{aligned}
& \mathrm{Es}_{i}=\mathrm{FA}_{i j} / \stackrel{\mathrm{FA}}{i j} \\
& \mathrm{Eh}_{j}=\stackrel{\mathrm{a}}{\mathrm{Es}} \mathrm{s}_{i j} \\
& \mathrm{ERh}_{j}=\mathrm{Eh}_{j} / \mathrm{a} \mathrm{Eh}_{j}
\end{aligned}
$$

Onde:

$\mathrm{i}=$ espécie $i$

$\mathrm{j}=$ hábitat $j$

$\mathrm{FA}_{i}=$ freqüência absoluta da espécie $i$ ou do gênero $i$

$\mathrm{Es}_{i}=$ especificidade da espécie $i$ ou do gênero $i$

$E h_{j}=$ especificidade do hábitat $j$

$\mathrm{ERh}_{j}=$ Especificidade relativa do hábitat $j$

Para a análise da similaridade da composição de espécies entre pares de estações amostrais foi utilizado o Índice de similaridade de Sørensen (IS) (Legendre \& Legendre, 1983):

$$
I S=2 a /[2 a+b+c]
$$

Onde:

IS = Índice de similaridade de Sørensen (x100) $\mathrm{a}=$ número de espécies em comum nas estações amostrais " $x$ " e " $y$ "

$b=$ número de espécies exclusivas da estação amostral " $x$ "

$\mathrm{C}=$ número de espécies exclusivas da estação amostral " $y$ "

Obs.: O resultado deste índice varia entre $0 \mathrm{a}$ $100 \%$.

Para a caracterização física e química da água foi realizado a determinação dos parâmetros $\mathrm{pH}$, condutividade e turbidez, foram feitas em campo com uma sonda multiparâmetros Horiba U10, enquanto que amostras de água foram coletadas e filtradas em campo para posterior determinação das concentrações de Amônio e Fosfato, no Laboratório de Oceanografia Química.

\section{RESULTADO E DISCUSSÃO}

As características físicas e químicas encontradas nessas águas apresentam um padrão consistente ao longo do gradiente longitudinal. Em levantamentos realizados pela equipe do Laboratório de Ecologia de Águas Interiores do CTTMar, verificou-se que nas estações amostrais localizadas mais à montante na bacia (estações 1 e 2), o pH é mais elevado $(7,3$ e 7,2$)$; e os valores de condutividade $(0,05$ e $0,06 \mathrm{mS} / \mathrm{cm})$, turbidez (13 e 8 NTU), amônio (5,05 e 4,32 mmol/L) e ortofosfato $(0,37$ e $0,3 \mathrm{mmol} / \mathrm{L})$ são mais baixos. As estações 3,4 e 5 apresentaram respectivamente, para estes mesmos parâmetros, $\mathrm{pH}$ mais baixo $(7,04 ; 6,93 ; 6,89)$; e condutividade $(0,0 ; 0,137 ; 0,244 \mathrm{mS} / \mathrm{cm})$, turbidez (16; 41; 40 NTU), amônio $(8,1 ; 33,0$; $76,7 \mathrm{mmol} / \mathrm{L})$ e ortofosfato $(0,53 ; 1,21 ; 3,7$ $\mathrm{mmol} / \mathrm{L}$ ) valores mais elevados (dados não publicados).

Foram encontradas 31 espécies pertencentes ao grupo das Chlorococcales (Chlorophyceae), representadas por 12 gêneros. O grupo das Chlorococcales foi mais abundante do que os outros grupos do fitoplâncton observados, como Heterokonthophyta (diatomáceas), Euglenophyta, Cyanobacteria, Dinophyta, e Conjugatophyceae.

Do total de espécies amostradas (Tabela 1), apenas 2 (Dictyosphaerium pulchellum e Pediastrum duplex) ocorreram em todas as estações amostrais (6,5\%), podendo ser consideradas como generalistas ou de ampla tolerância. Por outro lado, 9 espécies ocorreram em 2 estações amostrais e outras 13 ocorreram em apenas uma estação, somando $70,9 \%$ do total de espécies. As espécies com valores altos de especificidade $(1,0)$ podem ser consideradas como potenciais bioindicadores de qualidade ambiental.

Existe uma tendência à substituição de espécies nos hábitats, obedecendo a um gradiente das estações amostrais (Tabela 1). $\mathrm{Na}$ estação amostral 5 verificou-se a maior 
participação relativa de espécies exclusivas (33\%), entre as demais estações, e por outro lado, a estação 4 não apresentou espécies exclusivas.

$\mathrm{Na}$ estação 2 observou-se a maior riqueza específica (20), enquanto a menor foi verificada na estação 4 (Tabela 2). Esta estação não acompanhou um gradiente longitudinal do rio, indicando um provável estresse ambiental. Os resultados do índice de especificidade de hábitats variaram entre 2 (estação 4) e 10,5 (estação 2), sendo portanto 5 vezes maior, enquanto que a diferença na riqueza específica encontrada foi três vezes
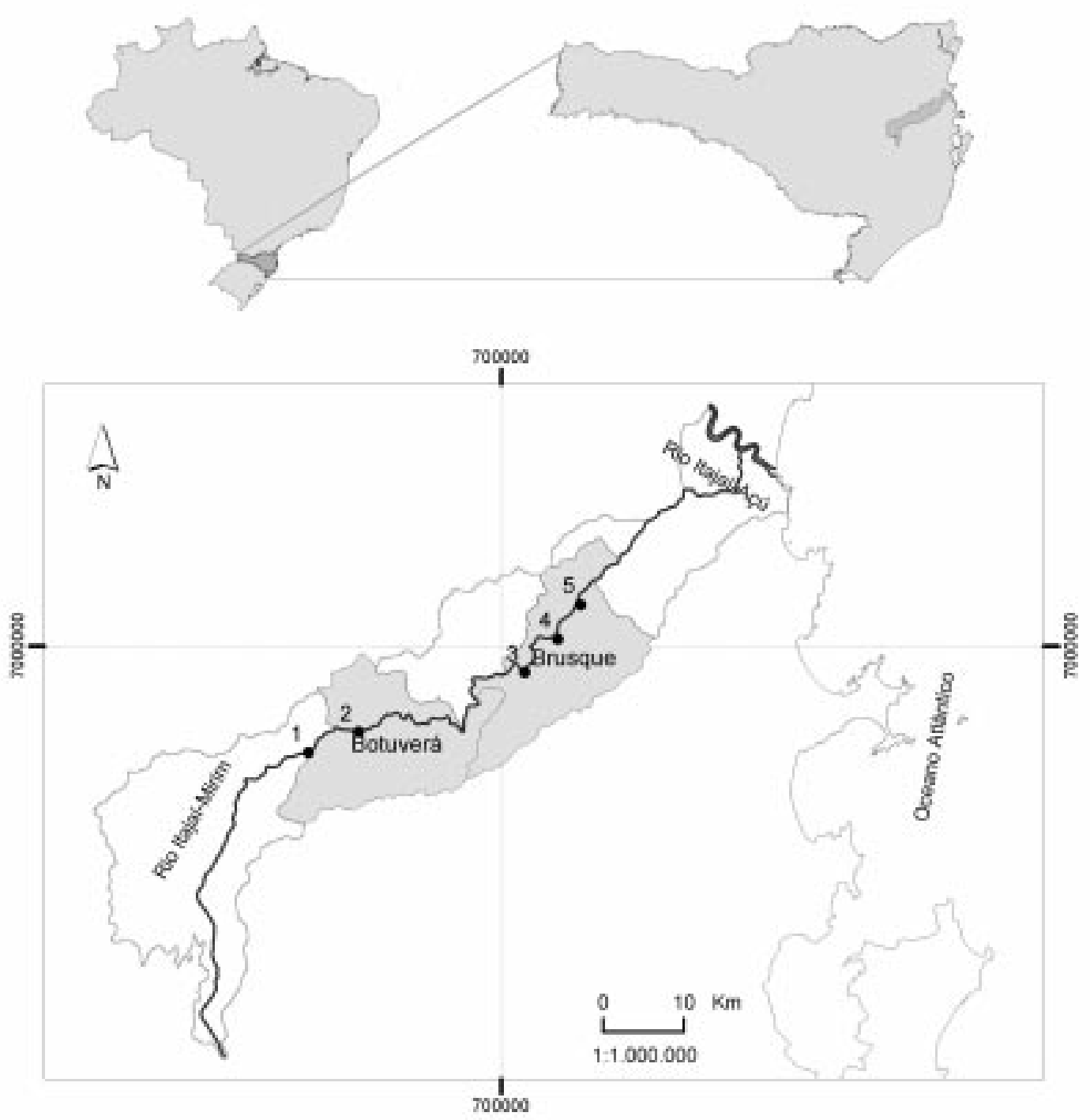

Figura 1. Localização das cinco estações amostrais no rio Itajaí-Mirim e as coordenadas UTM. 
Tabela 1 - Chlorococcales fitoplanctônicas encontradas nas 5 estações amostrais do rio Itajaí-Mirim, e valores de especificidade das espécies em relação ao hábitat.

\begin{tabular}{|c|c|c|c|c|c|}
\hline Espécies / estações amostrais & 1 & 2 & 3 & 4 & 5 \\
\hline Botryococcus sp. & & & & & 1 \\
\hline Kirchneriella dianae & & & & & 1 \\
\hline Pediastrum boryanum & & & & & 1 \\
\hline Scenedesmus smithii & & & & & 1 \\
\hline Coelastrum pulchrum & & & 0,5 & & 0,5 \\
\hline Scenedesmus longispina & & & 0,5 & & 0,5 \\
\hline Dictyosphaerium pulchellum & 0,2 & 0,2 & 0,2 & 0,2 & 0,2 \\
\hline Pediastrum duplex & 0,2 & 0,2 & 0,2 & 0,2 & 0,2 \\
\hline Coelastrum sphaericum & 0,25 & 0,25 & & 0,25 & 0,25 \\
\hline Crucigenia crucifera & 0,33 & 0,33 & & & 0,33 \\
\hline Scenedesmus quadricauda & & 0,33 & 0,33 & & 0,33 \\
\hline Scenedesmus tibiscensis & & 0,33 & 0,33 & & 0,33 \\
\hline Scenedesmus magnus & & 0,33 & 0,33 & 0,33 & \\
\hline Dimorphococcus lunatus & 0,25 & 0,25 & 0,25 & 0,25 & \\
\hline Nephrocytium cf. agardhianum & 0,25 & 0,25 & 0,25 & 0,25 & \\
\hline Scenedesmus opoliensis & & & 0,5 & 0,5 & \\
\hline Dictyosphaerium elegans & & & 1 & & \\
\hline Scenedesmus producto-capitatus & & & 1 & & \\
\hline Pediastrum simplex var. sturmii & & 1 & & & \\
\hline Pediastrum tetras & & 1 & & & \\
\hline Scenedesmus cf. pulloideus & & 1 & & & \\
\hline Scenedesmus disciformis & & 1 & & & \\
\hline Scenedesmus pecsensis & & 1 & & & \\
\hline Actinastrum rhaphidioides & 0,5 & 0,5 & & & \\
\hline Kirchneriella sp2 & 0,5 & 0,5 & & & \\
\hline Kirchneriella contorta & 0,5 & 0,5 & & & \\
\hline Micractinium sp. & 0,5 & 0,5 & & & \\
\hline Ooocystis sp. & 0,5 & 0,5 & & & \\
\hline Scenedesmus bernardii & 0,5 & 0,5 & & & \\
\hline Nephrocytium sp2 & 1 & & & & \\
\hline Scenedesmus cf. tenuispina & 1 & & & & \\
\hline
\end{tabular}


Tabela 2. Valores de riqueza e especificidade de espécies nos 5 hábitats estudados.

\begin{tabular}{lccccc}
\hline \multicolumn{1}{c}{ ESTAÇÕES } & $\mathbf{1}$ & $\mathbf{2}$ & $\mathbf{3}$ & $\mathbf{4}$ & $\mathbf{5}$ \\
\hline Riqueza específica & 14 & 20 & 12 & 7 & 12 \\
Índice de Especificidade do hábitat & 6,5 & 10,5 & 5,4 & 2,0 & 6,7 \\
Especificidade relativa do hábitat (\%) & 21 & 34 & 17 & 6 & 21 \\
\hline \hline
\end{tabular}

maior (Tabela 2). Este resultado demonstra que o índice de especificidade apresenta uma melhor resolução da contribuição proporcional de ocorrência das espécies no conjunto das estações amostrais.

Os resultados da especificidade relativa do hábitat apresentaram alta correlação com a riqueza específica (Correlação de Spearman $r_{s}=0,925$; Figura 2). Neste sentido, a especificidade relativa do hábitat tem como componente fundamental a própria riqueza e a proporção de ocorrência das espécies nos diferentes hábitats. Assim bastaria apenas utilizar a riqueza específica para indicar a especificidade do hábitat. Porém a alta correlação observada neste estudo pode ser atribuída à distribuição restrita das estações amostrais ao trecho médio do rio. Se fossem incluídos os hábitats mais extremos, como nascentes e foz, a especificidade tenderia ser maior. Constatou-se também que houve uma partição aproximadamente eqüitativa da riqueza de espécies nos grupos formados (exclusivas a generalistas) de especificidade de espécies e nas estações amostrais, somados a uma sobreposição gradual da composição específica ao longo do gradiente longitudinal do rio.

Verificou-se que as estações amostrais 4 e 5 possuem a maior similaridade na composição de espécies (Tabela 3). Logo a seguir, as estações 3 e 4 também demonstraram ser relativamente similares. Existe uma tendência de substituição das espécies desde as estações amostrais a montante (zonas mais preservadas) e a jusante (zonas mais perturbadas) do trecho estudado do rio, exceto para a estação 4 que se distancia deste padrão. Esta estação provavelmente é caracterizada por elevado nível de estresse (zona urbano-industrial) refletido na baixa riqueza biológica e na predominância de espécies de Chlorococcales com alta valência ecológica, inferida pelos baixos valores encontrados no índice de especificidade de espécies e do hábitat.

Os valores de riqueza e especificidade de gêneros nos 5 hábitats estudados são apresentados na Tabela 4. Verificou-se três grupos genéricos nítidos. As estações 3 e 4 apresentaram o menor valor e as estações $1 \mathrm{e}$ 2 os maiores valores (11 gêneros), levando-se em conta que o total das estações foi de 12

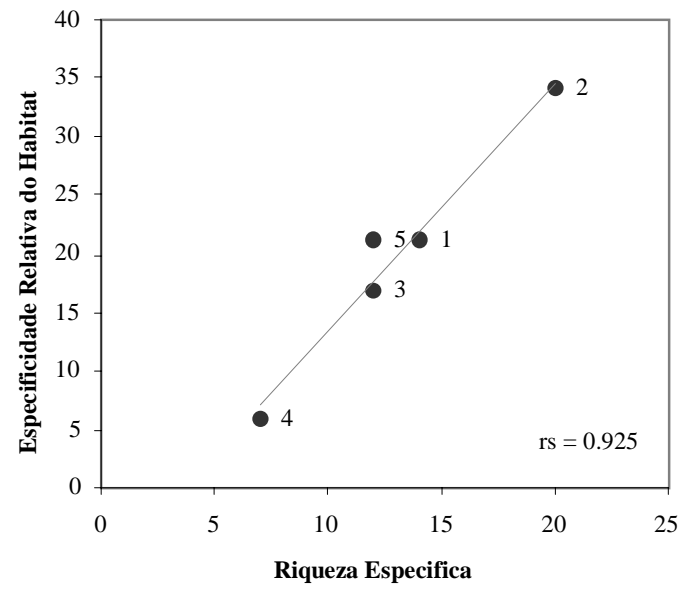

Figura 2 - Correlação entre Riqueza Específica e Especificidade Relativa do Hábitat das estações amostradas. Coeficiente de correlação de Spearman $\left(r_{s}\right)$ igual a 0,925 . 
Tabela 3 - Matriz de correlação dos Índices de Similaridade de Sorensen (\%) entre as estações amostrais com base na ocorrência das espécies.

\begin{tabular}{|c|ccccc|}
\hline Estações & $\mathbf{1}$ & $\mathbf{2}$ & $\mathbf{3}$ & $\mathbf{4}$ & $\mathbf{5}$ \\
\hline $\mathbf{1}$ & 100 & & & & \\
$\mathbf{2}$ & 71 & 100 & & & \\
$\mathbf{3}$ & 31 & 44 & 100 & & \\
$\mathbf{4}$ & 48 & 35 & 63 & 100 & \\
$\mathbf{5}$ & 31 & 38 & 58 & 40 & 100 \\
\hline
\end{tabular}

Tabela 4 - Valores de riqueza e especificidade de gêneros nos 5 hábitats estudados.

\begin{tabular}{lccccc}
\hline \multicolumn{1}{c}{ ESTAÇÕES } & $\mathbf{1}$ & $\mathbf{2}$ & $\mathbf{3}$ & $\mathbf{4}$ & $\mathbf{5}$ \\
\hline Riqueza genérica & 11 & 11 & 6 & 6 & 7 \\
Índice de Especificidade do hábitat & 3,5 & 3,5 & 1,3 & 1,3 & 2,5 \\
Especificidade relativa do hábitat $(\%)$ & 28,9 & 28,9 & 10,8 & 10,8 & 20,6 \\
\hline \hline
\end{tabular}

gêneros. Nesta escala, o índice de especificidade genérica demonstrou mais claramente os grupos de especificidade de hábitat. Inclusive com maior congruência com os resultados da análise de similaridade entre as estações amostrais.

\section{CONSIDERAÇÕES FINAIS}

Com base nos resultados obtidos, foi possível considerar 3 grupos de espécies. Um grupo com alta especificidade, composto por espécies com ocorrência exclusiva ou com ocorrência máxima em 2 hábitats; um segundo grupo com baixa especificidade, composto por espécies que ocorreram no mínimo em 3 hábitats. $O$ último grupo foi representado por espécies que ocorreram nos diversos hábitats (generalistas). Em relação à especificidade de hábitat, foram constatados três grupos, verificados também pelas especificidades de espécies e de gêneros, demonstrando uma tendência de distribuição dos hábitats ao longo do gradiente longitudinal no rio.

É necessário incorporar ao índice de especificidade medidas de outros atributos do hábitat ou mesmo da espécie, sendo inclusive previsto na proposição original de Wagner \& Edwards (2001). Os índices de especificidade utilizados neste trabalho deverão ser testados em outras situações ambientais e com outros grupos taxonômicos para validação da sua utilidade, com o intuito de elaborar critérios de classificação das espécies em relação às suas tolerâncias ambientais, no sentido de desenvolver ferramentas para diagnóstico ambiental.

\section{AGRADECIMENTOS}

Ao Conselho Nacional de Desenvolvimento Científico e Tecnológico 
(CNPq) pela concessão da bolsa RecémDoutor (Proc. 303716/03-7). Ao CTTMar por disponibilizar a logística e infra-estrutura para realização das campanhas e análises.

\section{REFERÊNCIAS BIBLIOGRÁFICAS}

Dufrene, M. \& P. Legendre. 1997. Species assemblages and indicator species: The need for a flexible asymmetrical approach. Ecological Monographs 67: 345-366.

Forman, R.T.T. 1995. Land mosaics: the ecology of landscapes and regions. Cambridge University Press. Cambridge, UK.

Komárek, J. \& B. Fott. 1983. Chlorophyceae (Grünalgen), Ordnung: Chlorococcales. In: Huber-Pestalozzi, G. (ed). Das Phytoplankton dês Süsswassers: Systematik und Biologie. Stuttgard: E. Schweizerbart'sche Verlagsbuchhandlung. 7 (1). 1044pp. PI. 1-253.

Kelly, M.G. \& B.A. Whitton. 1998. Biological monitoring of eutrophication in rivers. Hydrobiologia 384: 55-67.

Karr, J.R. \& E.W. Chu. 1999. Restoring life in Running Waters. Better Biological Monitoring. Island press. Washington, D.C. 206p.

Legendre, L. \& P. Legendre. 1983. Numerical Ecology. Elsevier Scientific Publishing Company. New York. 419p.
Riffel, E. \& A.C. Beaumord. 2002. Identificação das atividades antropogênicas potencialmente poluidoras do Rio ItajaíMirim e seus tributários no Município de Brusque, SC. Anais do Simpósio Brasileiro de Engenharia Ambiental. Itajaí, SC, Brasil.

Rosa, Z.M. \& A.L.B. Miranda-Kiesslich. 1988. O gênero Pediastrum Meyen (Chlorococcales-Hydrodictyaceae) do Sistema Lagunar da Região Litoral do Rio Grande do Sul, Brasil. Iheringia Sér. Bot. 38: 149-169. Porto Alegre.

Rosa, Z.M. \& A.L.B. Miranda-Kiesslich. 1989. Chlorococcales (Chlorophyceae) da Estação Ecológica do Taim, Rio Grande do Sul, Brasil. Insula 19: 215-228.

Silva, C.E. \& A.C. Beaumord. 2002. Caracterização das atividades de rizicultura e suas implicações ambientais na Bacia do Baixo Rio Itajaí-Mirim. Anais do Simpósio Brasileiro de Engenharia Ambiental. Itajaí, SC, Brasil.

Vannote, R.L.; Minshall, G.W.; Cummins, K.W.; Sedell, J.R. \& C.E. Cushing. 1989. The River Continnum Concept. Can. J. Fish. Aquat. Sci. 27: 130-137.

Wagner, H.H. \& P.J. Edwards. 2001. Quantifying habitat specificity to assess the contribution of a patch to species richness at a landscape scale. Landscape Ecology 16: 121-131. 